\title{
NICE-3-knockdown induces cell cycle arrest and autophagy in lung adenocarcinoma cells via the AKT/mTORC1 signaling pathway
}

\author{
LONGXIA DU ${ }^{1}$, YOURU WU ${ }^{1}$, XIAODAN HAN ${ }^{1}, \mathrm{CHEN} \mathrm{WANG}^{1}$, AILI LI $^{1}$ and GUOJIN HUANG ${ }^{1,2}$ \\ ${ }^{1}$ Laboratory of Respiratory Diseases, The Affiliated Hospital of Guilin Medical University; \\ ${ }^{2}$ Guangxi Key Laboratory of Molecular Medicine in Liver Injury and Repair, \\ Guilin Medical University, Guilin, Guangxi 541001, P.R. China
}

Received September 3, 2020; Accepted February 22, 2021

DOI: $10.3892 / \mathrm{etm} .2021 .10057$

\begin{abstract}
The NICE-3 protein serves an oncogenic role in hepatocellular carcinoma, but its role in lung adenocarcinoma (LUAD) remains unknown. The aim of the present study was to investigate the potential role and underlying mechanisms of NICE-3 in LUAD. In the present study, NICE-3 expression in LUAD tissues and its association with patient prognosis were analyzed using datasets from The Cancer Genome Atlas and Gene Express Omnibus. After NICE-3-knockdown with small interfering RNA in LUAD cells, cell proliferation was measured by cell counting, cell cycle was examined by flow cytometry, cell invasion and migration were detected by Transwell assays and autophagic markers LC3 and p62, as well as phosphorylation of S6K and AKT, were determined by western blotting. The results of public database analysis demonstrated that compared with normal lung tissues, NICE-3 expression was increased in LUAD tissues, where high expression levels were associated with a poor prognosis. The results of in vitro experimentation in LUAD cells indicated that NICE-3-knockdown inhibited proliferation, cell cycle, migration and invasion, but enhanced autophagy. Notably, NICE-3-knockdown inhibited AKT/mTORC1 signaling. The present results suggested that NICE-3 may serve an oncogenic role in LUAD via the AKT/mTORC1 signaling pathway and may therefore be a potential therapeutic target for LUAD.
\end{abstract}

\section{Introduction}

Lung cancer is one of the most life-threatening malignancies worldwide, accounting for $\sim 20 \%$ of all cancer-associated deaths

Correspondence to: Dr Guojin Huang, Laboratory of Respiratory Diseases, The Affiliated Hospital of Guilin Medical University, 15 Lequn Road, Guilin, Guangxi 541001, P.R. China E-mail: hgjj@163.com

Key words: NICE-3, chromosome 1 open reading frame 43, lung adenocarcinoma, proliferation, cell cycle, autophagy and with $>2$ million estimated new cases in 2018 (1). Lung cancer is classified as small cell lung cancer and non-small cell lung cancer, of which lung adenocarcinoma (LUAD) and lung squamous cell carcinoma are the most common subtypes (2). Although significant progress has been made in diagnosis and treatment during the last few decades (2), the prognosis of patients with LUAD remains poor. Therefore, the identification of novel molecules involved in the progression of LUAD may benefit the development of early diagnostic methods and/or targeted therapies.

AKT is a common component of multiple signaling pathways with a variety of downstream effectors (3), thereby integrating different upstream signals to regulate numerous cellular activities, including protein synthesis, migration and the cell cycle (3). The mechanistic target of rapamycin (mTOR) is one of the proteins downstream of AKT, and one of three core components of mTOR complex 1 (mTORC1). mTORC1 coordinates cellular proliferation and metabolism with nutrients and growth factors, as well as inhibits autophagy (4). Hyperactivation of AKT and mTORC1 has been observed in numerous types of cancer, including LUAD and colorectal carcinoma $(3,4)$.

The epidermal differentiation complex (EDC) is a gene cluster located on human chromosome 1q21 (5). Protein NICE-3 (also known as chromosome 1 open reading frame 43) is an EDC member originally identified from a keratinocyte cDNA library (5), which is expressed in the kidney and 25 other human tissues (6). NICE-3 primarily localizes to the Golgi and mitochondria, and interacts with plasma membrane proteins (7). Human histiocytic lymphoma U937 cells with NICE-3-deletion exhibit a strong defect in bacterial uptake (7), indicating its role as a regulator of phagocytosis (7). Furthermore, NICE-3 expression is upregulated in human hepatocellular carcinoma (HCC) and may contribute to HCC progression by promoting cellular proliferation, colony formation and the cell cycle (8). However, the detailed mechanisms underlying these phenomena remain unknown.

To the best of our knowledge, there are currently no studies on the function of NICE-3 in LUAD. Therefore, the aim of the current study was to investigate NICE-3 expression in LUAD tissues and its association with patient prognosis, as well as its function and associated underlying mechanisms. 


\section{Materials and methods}

Cell culture and transfection. The human LUAD A549 and H1993 cell lines were acquired from the Kunming Cell Bank of the Chinese Academy of Sciences (cat. KCB200434YJ; https://www.kmcellbank.com/index. php? $=$ content $\& a=$ show\&id=244) and the Chinese Tissue Culture Collections (cat. no. CTCC-007-0056; https://ctcc. online/), respectively. Cells were cultured in RPMI-1640 medium supplemented with $10 \%$ fetal bovine serum (FBS; both Gibco; Thermo Fisher Scientific, Inc.) and 1\% penicillin/streptomycin, and maintained in a humidified incubator at $37^{\circ} \mathrm{C}$ with $5 \% \mathrm{CO}_{2}$.

The sequences of the small interfering RNAs (siRNAs) targeting NICE-3 were as follows: si-NICE-3-\#1, 5'-CCU ACGGGAGCCUGGACUUTT-3'; si-NICE-3-\#2, 5'-GCUA UGAAACAGCCCGCUATT-3'; and si-NICE-3-\#3, 5'-GCU UGUGUCUAA AGGGUAATT-3'. The sequence of the non-targeting control siRNA (si-control) was 5'-UUCUCC GAACGUGUCACGUTT-3'. All siRNAs were synthesized by Shanghai GenePharma Co., Ltd. A549 cells (1.5x10 $/$ well) were seeded into six-well plates and cultured until $\sim 70 \%$ confluent. The cells were transfected at $37^{\circ} \mathrm{C}$ for $48 \mathrm{~h}$ with 75 pmol NICE-3 or control siRNA using Lipofectamine ${ }^{\circledR} 3000$ (Invitrogen; Thermo Fisher Scientific, Inc.) according to the manufacturer's instructions.

For autophagy assay, A549 and H1993 cells were transfected with si-NICE-3 or si-control for $48 \mathrm{~h}$ before being treated with the autophagy inhibitor bafilomycin A1 (100 nM; Targetmol) at room temperature for $1 \mathrm{~h}$, followed by western blotting assay.

Cell counting and colony formation assay. At $24 \mathrm{~h}$ post-transfection, A549 cells were harvested and manually counted under an optical microscope. The cells $\left(6 \times 10^{2} /\right.$ well $)$ were subsequently seeded into a 6-well plate, and cultured in RPMI 1640 medium (with 10\% FBS) in a humidified incubator at $37^{\circ} \mathrm{C}$ for $10-14$ days. The culture media were replaced every 2 days until the end of the experiment. Finally, the cells were fixed with $4 \%$ paraformaldehyde for $30 \mathrm{~min}$ at room temperature (RT), and then stained with $0.1 \%$ crystal violet at RT for $1 \mathrm{~h}$. The plates were dried and scanned using a Epson Perfection V370 Photo scanner. Cell colonies ( $>50$ cells) were counted manually.

Cell migration and invasion assays. The migration and invasion assays were performed as previously described (9). Transwell inserts (pore size, $8 \mu \mathrm{m}$ ) were coated with Matrigel at $37^{\circ} \mathrm{C}$ for $2 \mathrm{~h}$. A549 cells $\left(3 \times 10^{4}\right)$ were resuspended in $200 \mu \mathrm{l}$ serum-free RPMI medium and seeded into the upper chambers of the Transwell inserts (Costar; Corning Inc.). A total of $600 \mu 1$ RPMI medium supplemented with $20 \%$ FBS was added to the lower chambers. The cells were cultured at $37^{\circ} \mathrm{C}$ for $24 \mathrm{~h}$. The Transwell inserts were removed from the plate and the non-invasive or non-migratory cells were removed with a cotton swab. The migratory cells were fixed with $4 \%$ paraformaldehyde at room temperature for $15 \mathrm{~min}$ and stained with $0.1 \%$ crystal violet at $4^{\circ} \mathrm{C}$ overnight. Images of three randomly selected fields per well were captured using a TH4-200 light microscope (x200 magnification; Olympus Corporation).
Protein preparation and western blotting. Western blot analysis was conducted as previously described (10). At $48 \mathrm{~h}$ post-transfection, the cells were harvested and lysed in RIPA lysis buffer (Beijing Solarbio Science \& Technology Co., Ltd.) for $15 \mathrm{~min}$ on ice. The supernatants were collected before the protein concentration was determined using a bicinchoninic acid protein assay kit (Beyotime Institute of Biotechnology). The samples (30 $\mu \mathrm{g}$ protein) were resolved via 12 or $15 \%$ SDS-PAGE and transferred onto PVDF membranes, which were then blocked with 5\% non-fat milk at RT for $2 \mathrm{~h}$. The membranes were incubated with primary antibodies at $4^{\circ} \mathrm{C}$ overnight, washed three times with TBS-Tween $(0.1 \%)$ and then incubated with the secondary antibody at RT for $1 \mathrm{~h}$. The blots were washed and developed using BeyoECL Plus reagent (cat. no. P0018; Beyotime Institute of Biotechnology). The following primary antibodies were used: Anti-LC3B (cat. no. nb600-1384; 1:1,000) and anti-p62/sequestosome 1 (SQSTM1; cat. no. nbp1-48320; 1:1,000) purchased from Novus Biologicals, LLC; anti-phosphorylated (p)-AKT (cat. no. 4060; 1:1,000), anti-AKT (cat. no. 9272; 1:1,000), anti-p-S6K1 (cat. no. 9204; 1:1,000) and anti-S6K1 (cat. no. 9202; 1:1,000) purchased from Cell Signaling Technology, Inc.; mouse anti- $\beta$-actin antibody (cat. no. TA811000; $1: 1,000$ ) purchased from OriGene Technologies, Inc. The secondary antibodies were as follows: Horseradish peroxidase (HRP)-conjugated anti-mouse IgG (cat. no. 7076; Cell Signaling Technology, Inc.; 1:5,000) and HRP-conjugated anti-rabbit IgG (cat. no. 7074; Cell Signaling Technology, Inc.; 1:5,000). Densitometric analysis was performed using ImageJ software (v1.53a; National Institutes of Health).

Flow cytometry. A549 cells were collected $48 \mathrm{~h}$ after transfection and washed three times with pre-cooled PBS. The cells were then fixed with $70 \%$ ethanol overnight at $4^{\circ} \mathrm{C}$, followed by a further three washes with cold PBS. The cells $\left(2 \times 10^{5}\right)$ were stained with the staining solution $(20 \mu \mathrm{g} / \mathrm{ml}$ propidium iodide and $200 \mu \mathrm{g} / \mathrm{ml}$ RNase; cat. no. F10797; Thermo Fisher Scientific, Inc.) and then incubated at RT for $1 \mathrm{~h}$ in a dark chamber. The cell cycle was analyzed by flow cytometry (NovoCyte 2060R; ACEA Biosciences, Inc.) with Software NovoExpress 1.4.0 (ACEA Biosciences, Inc.).

Analysis of NICE-3 expression and patient prognosis in LUAD. NICE-3 expression analysis in the LUAD dataset from The Cancer Genome Atlas (TCGA) was conducted using UALCAN (11). The expression of NICE-3 in lung adenocarcinoma tissue and normal lung tissue from the GSE31210 microarray dataset (12) on Gene Expression Omnibus database (https://www.ncbi.nlm.nih.gov/geo/query/acc. cgi?acc=GSE31210) was analyzed using GraphPad Prism 5.0 (GraphPad Software, Inc.). The effects of NICE-3 expression on overall survival (OS), first progression (FP) and post-progression survival (PPS) were determined using the Kaplan-Meier Plotter online tool (www.kmplot.com) (13). The cut-off values were automatically determined by the software. Log-rank test was used to evaluate the differences between survival curves. The Cox proportional hazards model in the Kaplan-Meier Plotter online tool was used for multivariate analysis of the effects of clinical characteristics and NICE-3 expression on OS and FP. 
Statistical analysis. All statistical analyses were conducted using GraphPad Prism 8 (GraphPad Software, Inc.). All experiments were repeated $\geq$ three times independently. Values are expressed as the mean \pm standard deviation. Unpaired Student's t-test was used for comparisons between two groups, and ANOVA followed by Tukey's post-hoc test was used for comparisons among multiple groups. $\mathrm{P}<0.05$ was considered to indicate a statistically significant difference.

\section{Results}

NICE-3 expression is increased in LUAD tissues, and high expression is associated with poor survival outcomes. The UALCAN online tool was used to determine NICE-3 expression in TCGA LUAD dataset (11). The results revealed that NICE-3 expression was significantly increased in LUAD compared with in normal lung samples $(\mathrm{P}<0.0001$; Fig. 1A). To confirm this finding, the GSE31210 dataset from the Gene Expression Omnibus database was also analyzed (12). The results also demonstrated that NICE-3 expression was significantly upregulated in LUAD compared with in normal lung samples $(\mathrm{P}<0.01$; Fig. 1B), consistent with the results of TCGA LUAD dataset.

In order to assess the prognostic value of NICE-3 in patients with LUAD, the associations between NICE-3 expression and OS, FP and PPS were analyzed using the Kaplan-Meier plotter (13). The patients were separated into low and high NICE-3 expression groups according to the cut-off values determined by the software (177 for OS, 178 for FP and 148 for PPS). The results suggested that high NICE-3 expression was significantly associated with a poorer OS and FP compared with low NICE-3 expression, while no significant association with PPS was observed (Fig. 2), implying that NICE-3 expression may serve as a predictive indicator of OS and FP. Furthermore, the results of the multivariate analysis of clinical characteristics, such as American Joint Committee on Cancer stage (14), sex and smoking history, suggested that high NICE-3 expression may be an independent prognostic indicator for OS only (Table I). In addition, the results indicated that AJCC stage $\mathrm{T}$ associated significantly with OS and FP (Table I).

NICE-3-knockdown inhibits the proliferation, cell cycle progression, migration and invasion of LUAD cells. To determine how NICE-3 negatively impacts patient prognosis, the LUAD A549 cell line was used to perform in vitro experiments. A total of three siRNA molecules specifically targeting NICE-3 (si-NICE-3) were designed, and their efficacy was confirmed in A549 and H1993 cells transfected with si-NICE-3 or si-control $48 \mathrm{~h}$ after transfection. NICE-3 protein expression was subsequently detected by western blotting. The blots demonstrated that si-NICE-3-\#3 exerted the strongest inhibitory effect on NICE-3 protein expression and was thus selected for subsequent experimentation (Fig. 3A).

To investigate the effects of NICE-3-knockdown on cellular proliferation, A549 and H1993 cells were transfected with si-NICE-3 and counted $24 \mathrm{~h}$ later. The results revealed that compared with the control group, NICE-3-knockdown significantly decreased cell numbers (Fig. 3B), indicating that cellular proliferation was impaired by NICE-3-knockdown.
Table I. Cox regression multivariate analysis of NICE-3 expression and OS or FP in patients with lung adenocarcinoma.

\begin{tabular}{lcc} 
A, OS & & \\
\hline Variable & Hazard ratio (95\% CI) & P-value \\
\hline Sex & $1.39(0.74-2.61)$ & 0.3005 \\
Stage & $2.88(0.48-17.13)$ & 0.2453 \\
AJCC T stage & $3.08(1.36-6.98)$ & $0.0069^{\text {a }}$ \\
AJCC N stage & $0.91(0.15-5.36)$ & 0.9178 \\
Smoking history & $0.85(0.36-1.97)$ & 0.6988 \\
NICE-3 expression & $0.51(0.27-0.94)$ & $0.0305^{\text {b }}$ \\
\hline
\end{tabular}

B, FP

\begin{tabular}{lcc}
\hline Variable & Hazard ratio $(95 \% \mathrm{CI})$ & P-value \\
\hline Sex & $1.24(0.62-2.48)$ & 0.5460 \\
Stage & $0.93(0.08-10.32)$ & 0.9506 \\
AJCC T stage & $4.24(1.48-12.18)$ & $0.0073^{\text {a }}$ \\
AJCC N stage & $2.33(0.22-24.94)$ & 0.4829 \\
Smoking history & $1.55(0.69-3.48)$ & 0.2863 \\
NICE-3 expression & $0.58(0.29-1.16)$ & 0.1265
\end{tabular}

${ }^{\mathrm{a}} \mathrm{P}<0.01 ;{ }^{\mathrm{b}} \mathrm{P}<0.05$. AJCC, American Joint Committee on Cancer; $\mathrm{T}$, tumor; N, node; OS, overall survival; FP, first progression.
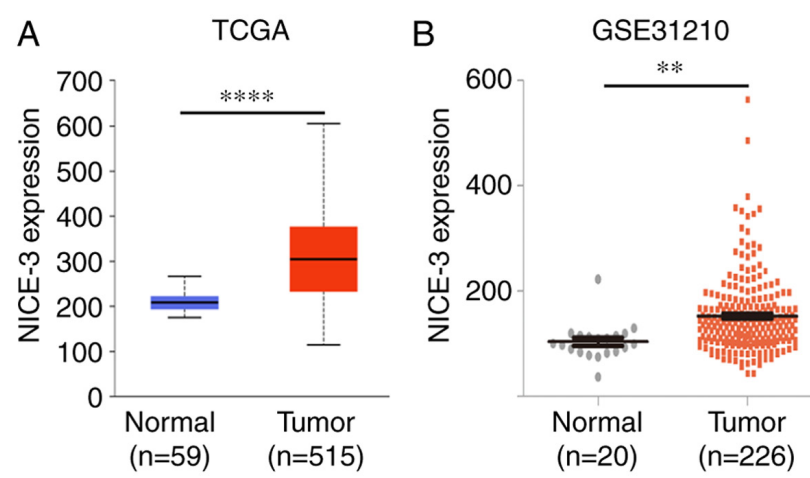

Figure 1. NICE-3 expression is increased in LUAD. (A) NICE-3 expression in TCGA LUAD samples $(n=515)$ and normal lung samples $(n=59)$ was analyzed using the UALCAN online database. NICE-3 expression was significantly upregulated in LUAD samples. (B) NICE-3 expression in GSE31210 LUAD tissues ( $\mathrm{n}=226$ ) and normal lung tissues $(\mathrm{n}=20)$ was analyzed using GraphPad Prism. NICE-3 expression was significantly upregulated in LUAD. ${ }^{* *} \mathrm{P}<0.01$; ${ }^{* * * *} \mathrm{P}<0.0001$. LUAD, lung adenocarcinoma; TCGA, The Cancer Genome Atlas.

A colony formation assay was performed to assess the effects of NICE-3 on colony formation ability following NICE-3-knockdown. The results demonstrated that NICE-3-knockdown significantly compromised colony formation capacity (Fig. 3C), suggesting that NICE-3 may be involved in cellular survival.

In order to elucidate how cellular proliferation and survival are influenced by NICE-3, the A549 cell cycle was analyzed via flow cytometry following NICE-3-knockdown. The results demonstrated that compared with the si-control, 
OS

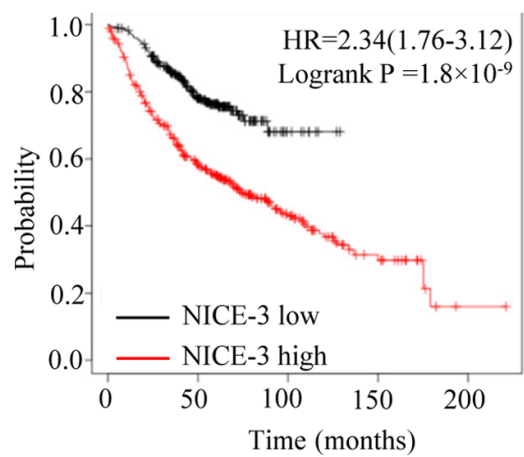

FP

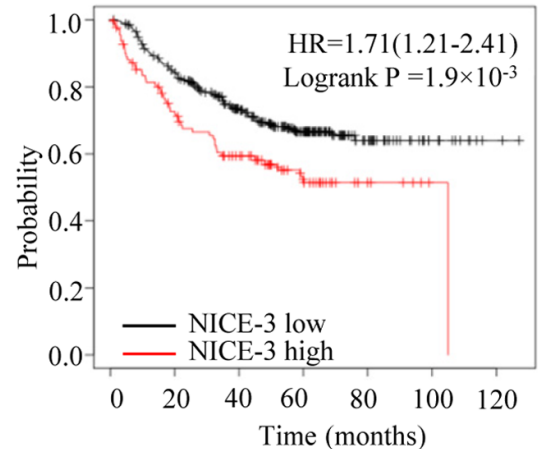

PPS

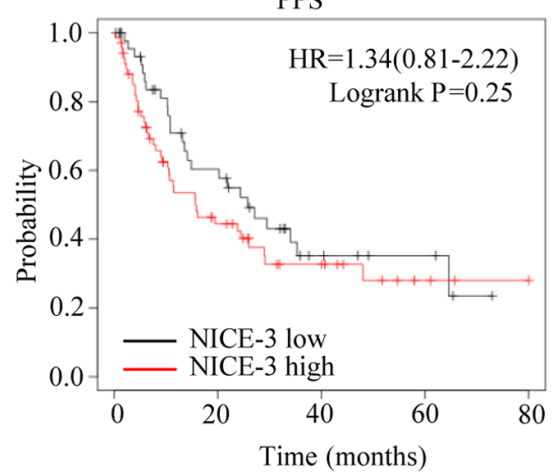

Figure 2. High NICE-3 expression is associated with poor OS and FP in patients with lung adenocarcinoma. High NICE-3 expression was associated with poor OS $(n=672)$ and FP $(n=443)$. High NICE-3 expression was not significantly associated with PPS $(n=35)$. Survival plots were generated using the Kaplan-Meier Plotter. OS, overall survival; FP, first progression; PPS, post-progression survival; HR, hazard ratio.

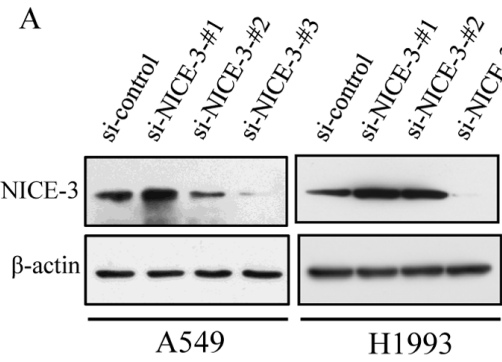

$\mathrm{C}$

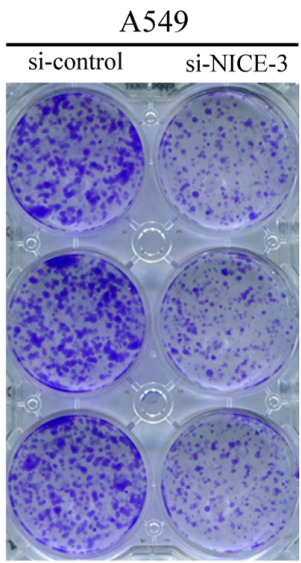

D

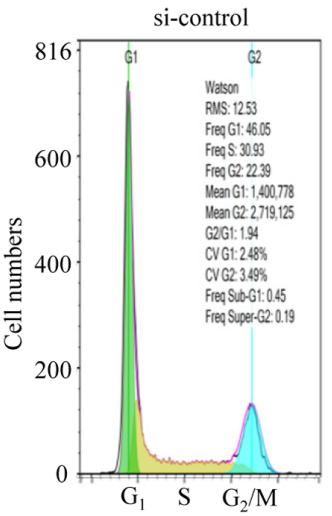

H1993

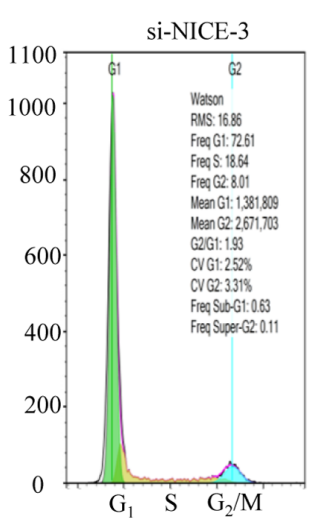

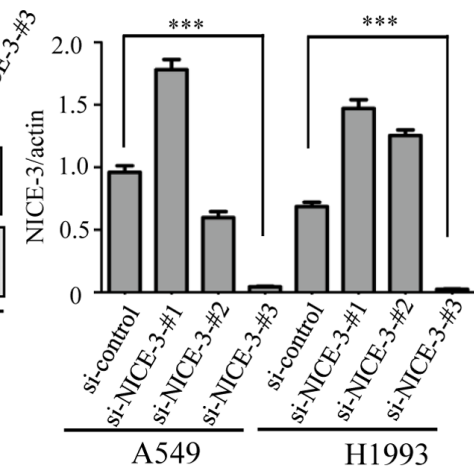
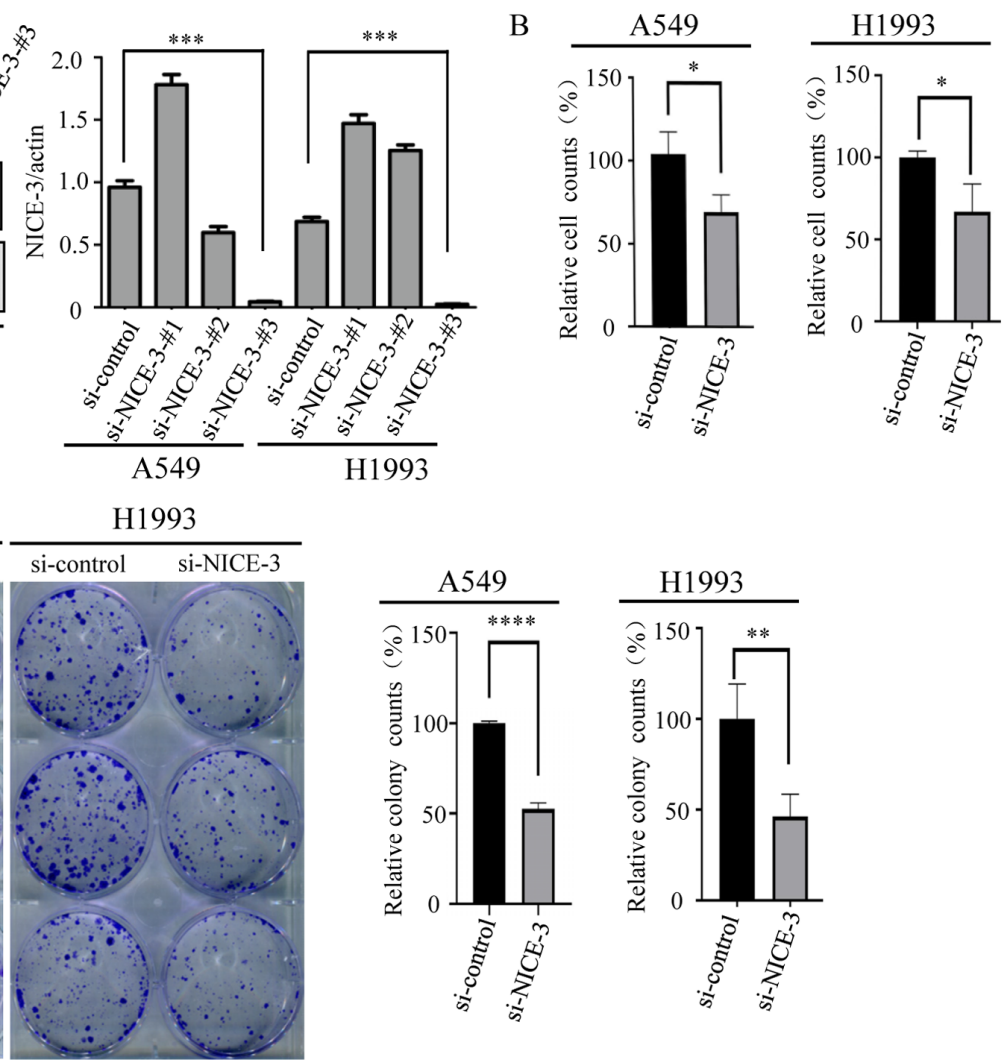

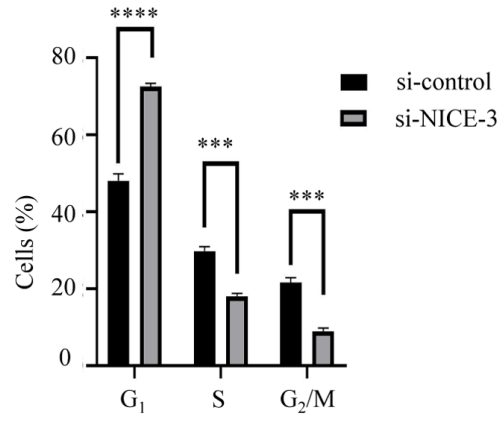

Figure 3. NICE-3-knockdown inhibits cellular proliferation, colony formation and the cell cycle. (A) A549 cells were transfected with si-NICE-3 for $48 \mathrm{~h}$. NICE-3 protein expression was then assessed by western blotting, indicating that si-NICE-3-\#3 exerted the greatest inhibitory effect. (B) A549 and H1993 cells were transfected with si-NICE-3 and proliferation was assessed by cell counting. NICE-3-knockdown suppressed the proliferation of A549 and H1993 cells. (C) Colony formation assay results of A549 and H1993 cells transfected with si-NICE-3 or si-control. (D) Cell cycle analysis results of A549 cells transfected with si-NICE-3 or si-control. ${ }^{*} \mathrm{P}<0.05 ;{ }^{* * *} \mathrm{P}<0.01 ;{ }^{* * * *} \mathrm{P}<0.001 ;{ }^{* * * * * *} \mathrm{P}<0.0001$. si, small interfering RNA. 

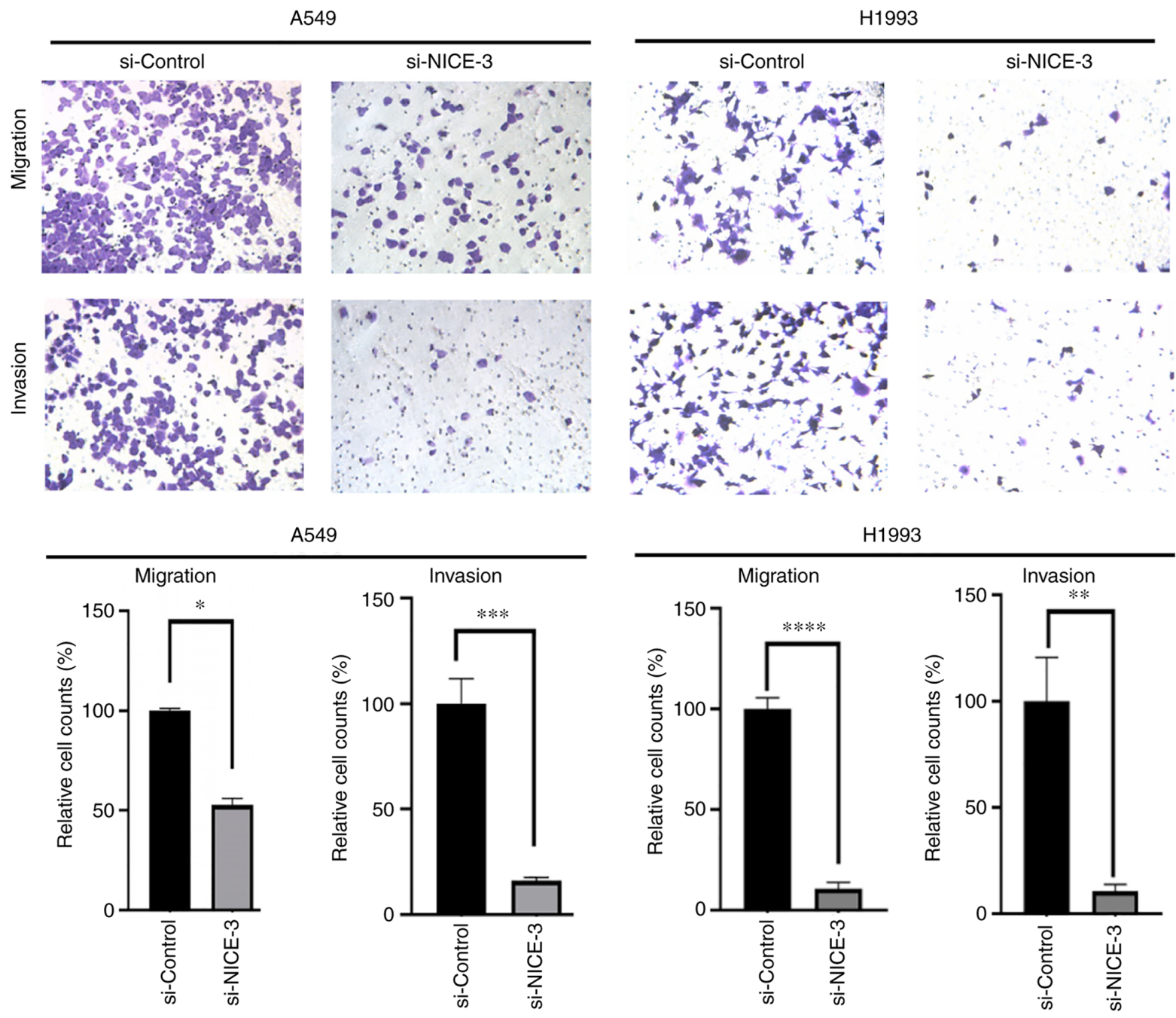

Figure 4. NICE-3-knockdown suppresses the migration and invasion of lung adenocarcinoma cells. Migration and invasion of A549 and H1993 cells transfected with si-NICE-3 or si-control were assessed using Transwell assays without or with Matrigel, respectively. Representative images are shown (magnification, $\mathrm{x} 200)$. ${ }^{*} \mathrm{P}<005 ;{ }^{* *} \mathrm{P}<0.01 ;{ }^{* * *} \mathrm{P}<0.001 ;{ }^{* * * * *} \mathrm{P}<0.0001$. si, small interfering RNA.

si-NICE-3 induced $\mathrm{G}_{1}$ phase arrest (Fig. 3D), indicating that NICE-3 positively regulated the cell cycle, and thus enhanced cellular proliferation and survival. The H1993 cell cycle was also analyzed via flow cytometry following NICE-3 knockdown, but no significant effect was observed (data not shown).

To investigate the role of NICE-3 in cellular migration and invasion, A549 and H1993 cells were transfected with si-NICE-3 or si-control prior to Transwell migration and invasion assays, respectively. The results demonstrated that NICE-3-knockdown significantly inhibited the migration and invasion of A549 and H1993 cells (Fig. 4), suggesting that NICE-3 may be positively associated with the migration and invasion of LUAD cells.

NICE-3-knockdown promotes autophagy in LUAD cells. To investigate the potential role of NICE-3 in autophagy, A549 and $\mathrm{H} 1993$ cells were transfected with si-NICE-3 or si-control for $48 \mathrm{~h}$, and then treated with the autophagy inhibitor bafilomycin A1 (100 nM) for $1 \mathrm{~h}$. The autophagic marker proteins LC3B and p62/SQSTM1 were then detected by western blot analysis (15). The results indicated that NICE-3-knockdown promoted the conversion of LC3-I to LC3-II, as well as the degradation of p62/SQSTM1 (Fig. 5A). Following treatment with bafilomycin A1, LC3-II and p62/SQSTM1 had notably accumulated in the si-NICE-3 group compared with in the control group (Fig. 5A). These findings suggested that NICE-3 inhibited A549 and H1993 cell autophagy.

NICE-3-knockdown inhibits AKT/mTORCl signaling in LUAD cells. To investigate the underlying mechanisms by which NICE-3 may regulate autophagy, A549 and H1993 cells were transfected with si-NICE-3 or si-control for $48 \mathrm{~h}$, and then the levels of AKT, p-AKT, p70 S6K and p-p70 S6K were detected by western blotting. The results revealed that NICE-3-knockdown significantly inhibited the phosphorylation of AKT and p70 S6K without influencing total AKT and p70 S6K protein levels (Fig. 5B), suggesting that NICE-3 may function through the $\mathrm{AKT} / \mathrm{mTORC} 1$ signaling pathway.

\section{Discussion}

NICE-3 is a rarely studied molecule, which was first identified from a keratinocyte cDNA library by Marenholz et al (5) in 
A
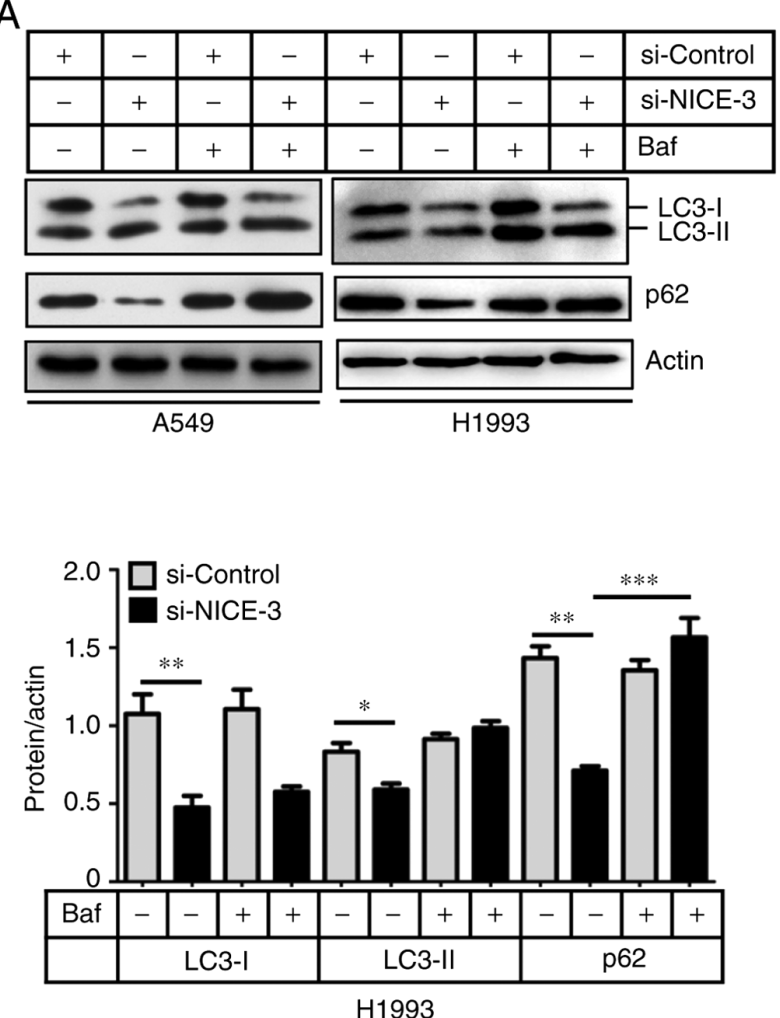
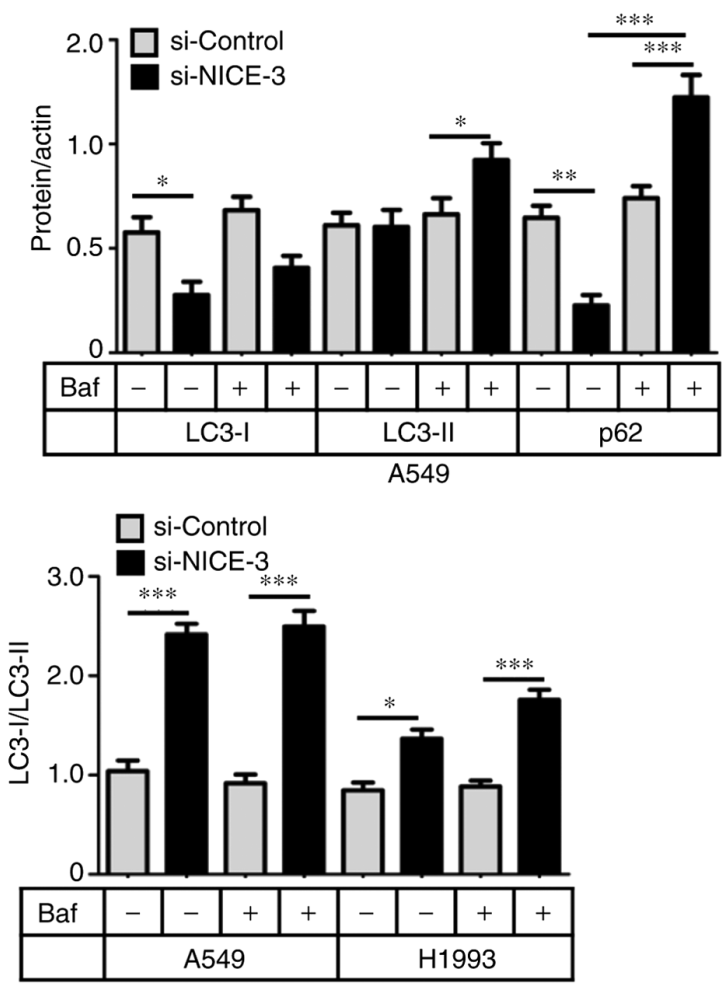

$\mathrm{B}$
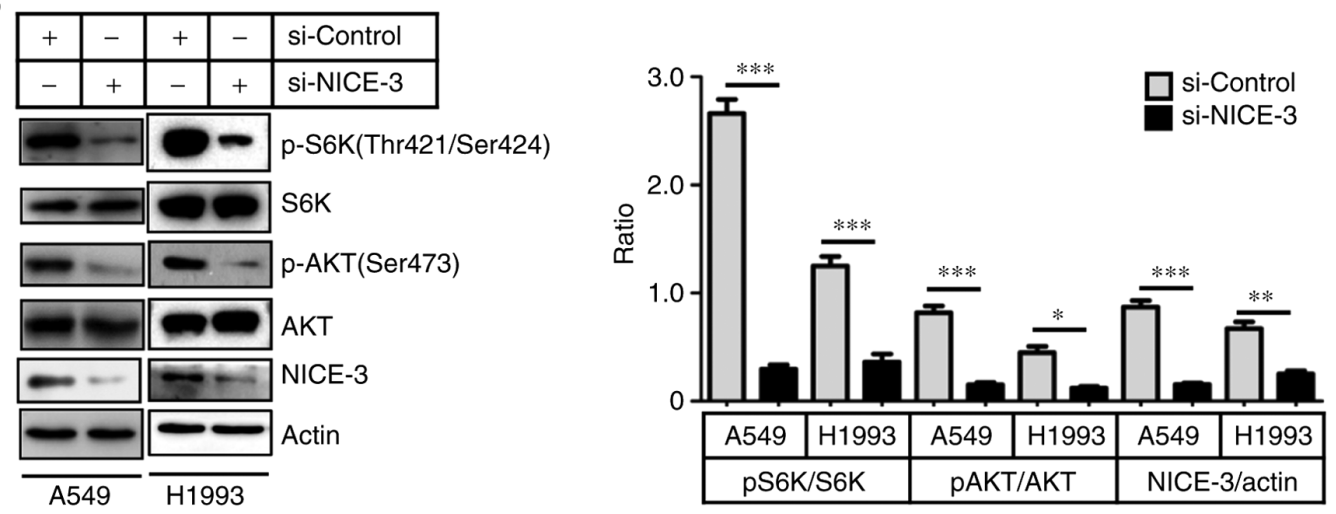

Figure 5. NICE-3-knockdown induces autophagy by inhibiting the AKT/mTORC1 signaling pathway. A549 and H1993 cells were transfected with si-NICE-3 or si-control for $48 \mathrm{~h}$, followed by bafilomycin treatment for $1 \mathrm{~h}$. LC3 and p62 proteins, as well as AKT and S6K phosphorylation, were evaluated by western blot analysis. (A) Conversion of LC3-I to LC3-II (LC3-II/LC3-I ratio as an indicator) was enhanced, and p62 protein levels were decreased by si-NICE-3, indicating increased autophagy. Representative western blots are shown. The bands were quantified using ImageJ and normalized to the loading control. (B) Phosphorylation of AKT and S6K were suppressed by NICE-3-knockdown, indicating decreased AKT and mTORC1 activity. Representative western blots are shown. The bands were quantified using ImageJ and normalized to the loading control. ${ }^{*} \mathrm{P}<005 ;{ }^{* *} \mathrm{P}<0.01 ;{ }^{* * *} \mathrm{P}<0.001$. si, small interfering RNA; mTORC1, mechanistic target of rapamycin complex 1; Baf, bafilomycin; $\mathrm{p}$, phosphorylated.

2001. After over a decade, Wei et al (8) reported that NICE-3 expression was upregulated in HCC and was involved in cellular proliferation, colony formation and the cell cycle. However, the potential role that NICE-3 serves in other types of cancer remained unclear. By analyzing its expression levels in two LUAD datasets, the present study revealed that NICE-3 expression was increased in LUAD, and that high expression levels were associated with poor OS and FP. The current findings indicated that NICE-3 may be a potential prognostic marker in LUAD.

Excessive proliferation is a typical feature of malignant tumor growth (16). Since NICE-3 was found to promote cell cycle progression in HCC (8), the present study hypothesized that inhibiting NICE-3 expression may inhibit the cell cycle in LUAD cells. Therefore, the effects of NICE-3 expression were assessed in vitro using LUAD cells. The results demonstrated that NICE-3-knockdown in A549 cells inhibited cellular proliferation and induced cell cycle arrest, consistent with previous observations in HCC (8). Cellular migration and invasion are essential features for tumor metastasis (16). The results of the present study indicated that NICE-3-knockdown inhibited A549 and H1993 cell migration and invasion, suggesting that NICE-3 may be involved in tumor metastasis.

Autophagy is a conserved pathway through which damaged proteins and organelles are degraded to maintain 
cellular homeostasis or in response to extracellular cues (17). Previous studies have revealed that autophagy may function as a tumor-suppressing or -promoting process, depending on the type of tumor or context (18). Becnl heterozygous-deficient mice are prone to the development of liver and lung tumors $(19,20)$. Basal autophagy is upregulated in hypoxic tumor regions of cervical cancer where it is essential for cell survival (21). Wei et al (15) reported that autophagy inhibition was associated with increased clonogenic survival of non-small cell lung cancer cells. Thus, the role of NICE-3 on autophagy was investigated in the current study, revealing that NICE-3 may inhibit autophagy.

Although NICE-3 serves an oncogenic role in HCC (8), the associated underlying mechanisms remain unknown. The results of the present study revealed a novel mechanism by which NICE-3-knockdown decreased AKT and S6K phosphorylation, indicating that NICE-3 positively regulated AKT and mTORC1 activity in LUAD cells, and thus enhanced AKT/mTORC1 signaling. Since AKT promotes cellular proliferation, motility and cell cycle progression $(3,22)$, NICE-3-knockdown induced cell cycle arrest, attenuated cellular proliferation and suppressed the migration and invasion of LUAD cells. To the best of our knowledge, the present study was the first to reveal the association between NICE-3 expression, the AKT/mTORC1 signaling pathway and autophagy.

In the present study, in vitro experimentation was conducted by knocking down NICE-3 expression in A549 and H1993 cells. Therefore, in order to confirm the present findings, further experiments, including NICE-3 overexpression and knockdown, should be conducted in other lung cancer cell lines. In addition, in vivo LUAD tumor xenograft experiments are required to confirm the present in vitro findings. Since autophagy was only examined by detecting LC3-I/II and p62 protein levels using western blotting, further experiments should be performed in the future to monitor autophagy using additional techniques, such as transmission electron microscopy and GFP-LC3 fluorescence microscopy, to confirm the effect of NICE-3 on autophagy.

In conclusion, the results of the current study revealed that NICE-3 expression was increased in LUAD tissues, and that high expression levels were associated with a poor prognosis. Furthermore, NICE-3 promoted the proliferation, cell cycle progression, invasion and migration of LUAD cells, as well as suppressed autophagy. Therefore, NICE-3 may serve an oncogenic role in LUAD and may be a potential therapeutic target.

\section{Acknowledgements}

Not applicable.

\section{Funding}

The present study was supported by the Hundred Talents Program of Guangxi, Natural Science Foundation of Guangxi (grant no.2020GXNSFAA297209), the ResearchEnhancement Project for Junior Faculty in Higher Education Institutes of Guangxi (grant no. 2019KY0522), the Open Research Fund from Guangxi Key Laboratory of Liver Injury and Repair Molecular Medicine (grant no. GXLIRMMKL-201802 and GXLIRMMKL-201816) and the Scientific Research
Project for Junior Faculty in Guilin Medical College (grant no. 2018glmcy055).

\section{Availability of data and materials}

The datasets used and/or analyzed during the current study are available from the corresponding author on reasonable request.

\section{Authors' contributions}

GH designed and supervised the study. LD, YW, XH, CW and AL conducted the experiments and collected the data. LD and $\mathrm{GH}$ confirm the authenticity of all the raw data. LD and GH interpreted the data and drafted the initial manuscript. All authors read and approved the final version of the manuscript.

\section{Ethics approval and consent to participate}

Not applicable.

\section{Patient consent for publication}

Not applicable.

\section{Competing interests}

The authors declare that they have no competing interests.

\section{References}

1. Bray F, Ferlay J, Soerjomataram I, Siegel RL, Torre LA and Jemal A: Global cancer statistics 2018: GLOBOCAN estimates of incidence and mortality worldwide for 36 cancers in 185 countries. CA Cancer J Clin 68: 394-424, 2018.

2. Herbst RS, Morgensztern D and Boshoff C: The biology and management of non-small cell lung cancer. Nature 553: 446-454, 2018.

3. Manning BD and Toker A: AKT/PKB signaling: Navigating the network. Cell 169: 381-405, 2017.

4. Saxton RA and Sabatini DM: mTOR signaling in growth, metabolism, and disease. Cell 168: 960-976, 2017.

5. Marenholz I, Zirra M, Fischer DF, Backendorf C, Ziegler A and Mischke D: Identification of human epidermal differentiation complex (EDC)-encoded genes by subtractive hybridization of entire YACs to a gridded keratinocyte cDNA library. Genome Res 11: 341-355, 2001

6. Fagerberg L, Hallström BM, Oksvold P, Kampf C, Djureinovic D, Odeberg J, Habuka M, Tahmasebpoor S, Danielsson A, Edlund K, et al: Analysis of the human tissue-specific expression by genome-wide integration of transcriptomics and antibody-based proteomics. Mol Cell Proteomics 13: 397-406, 2014.

7. Jeng EE, Bhadkamkar V, Ibe NU, Gause H, Jiang L, Chan J, Jian R, Jimenez-Morales D, Stevenson E, Krogan NJ, et al: Systematic identification of host cell regulators of legionella pneumophila pathogenesis using a genome-wide CRISPR Screen. Cell Host Microbe 26: 551-563.e6, 2019.

8. Wei YJ,Hu QQ, Gu CY, Wang YP,Han ZG and Cai B: Up-regulation of NICE-3 as a novel EDC gene could contribute to human hepatocellular carcinoma. Asian Pac J Cancer Prev 13: 4363-4368, 2012.

9. Wang W, Li A,Han X, Wang Q, Guo J, Wu Y, Wang C and Huang G: DEPDC1 up-regulates RAS expression to inhibit autophagy in lung adenocarcinoma cells. J Cell Mol Med 24: 13303-13313, 2020.

10. Li A, Wang Q, He G, Jin J and Huang G: DEP domain containing 1 suppresses apoptosis via inhibition of A20 expression, which activates the nuclear factor $\kappa \mathrm{B}$ signaling pathway in HepG2 cells. Oncol Lett 16: 949-955, 2018.

11. Chandrashekar DS, Bashel B, Balasubramanya SAH, Creighton CJ, Ponce-Rodriguez I, Chakravarthi BVSK and Varambally S: UALCAN: A portal for facilitating tumor subgroup gene expression and survival analyses. Neoplasia 19: 649-658, 2017. 
12. Okayama H, Kohno T, Ishii Y, Shimada Y, Shiraishi K, Iwakawa R, Furuta K, Tsuta K, Shibata T, Yamamoto S, et al: Identification of genes upregulated in ALK-positive and EGFR/KRAS/ALK-Negative lung adenocarcinomas. Cancer Res 72: 100-111, 2012

13. Győrffy B, Surowiak P, Budczies J and Lánczky A: Online survival analysis software to assess the prognostic value of biomarkers using transcriptomic data in non-small-cell lung cancer. PLoS One 8: e82241, 2013.

14. Edge SB, Byrd DR, Compton CC, Fritz AG, Greene FL and Trotti A (eds): AJCC Cancer Staging Manual. 7th edition. Springer, New York, NY, 2010.

15. Wei Y, Zou Z, Becker N, Anderson M, Sumpter R, Xiao G, Kinch L, Koduru P, Christudass CS, Veltri RW, et al: EGFR-mediated beclin 1 phosphorylation in autophagy suppression, tumor progression, and tumor chemoresistance. Cell 154: 1269-1284, 2013.

16. Hanahan D and Weinberg RA: Hallmarks of cancer: The next generation. Cell 144: 646-674, 2011.

17. Levine B and Kroemer G: Biological functions of autophagy genes: A disease perspective. Cell 176: 11-42, 2019.

18. White E: The role for autophagy in cancer. J Clin Invest 125 : $42-46,2015$
19. Qu X, Yu J, Bhagat G, Furuya N, Hibshoosh H, Troxel A, Rosen J, Eskelinen EL, Mizushima N, Ohsumi Y, et al: Promotion of tumorigenesis by heterozygous disruption of the beclin 1 autophagy gene. J Clin Invest 112: 1809-1820, 2003.

20. Yue Z, Jin S, Yang C, Levine AJ and Heintz N: Beclin 1, an autophagy gene essential for early embryonic development, is a haploinsufficient tumor suppressor. Proc Natl Acad Sci USA 100: 15077-15082, 2003.

21. Degenhardt K, Mathew R, Beaudoin B, Bray K, Anderson D, Chen G, Mukherjee C, Shi Y, Gélinas C, Fan Y, et al: Autophagy promotes tumor cell survival and restricts necrosis, inflammation, and tumorigenesis. Cancer Cell 10: 51-64, 2006.

22. Chin YR and Toker A: Function of Akt/PKB signaling to cell motility, invasion and the tumor stroma in cancer. Cell Signal 21: 470-476, 2009

This work is licensed under a Creative Commons Attribution-NonCommercial-NoDerivatives 4.0 International (CC BY-NC-ND 4.0) License. 